\title{
BMJ Open Changing adherence-related beliefs about ICS maintenance treatment for asthma: feasibility study of an intervention delivered by asthma nurse specialists
}

\author{
Sarah C E Chapman, ${ }^{1}$ Neil Barnes, ${ }^{2}$ Mari Barnes, ${ }^{3}$ Andrea Wilkinson, ${ }^{1}$ \\ John Hartley, ${ }^{4}$ Cher Piddock, ${ }^{5}$ John Weinman, ${ }^{6}$ Rob Horne ${ }^{1}$
}

To cite: Chapman SCE, Barnes N, Barnes M, et al. Changing adherence-related beliefs about ICS maintenance treatment for asthma: feasibility study of an intervention delivered by asthma nurse specialists. BMJ Open 2015;5:e007354. doi:10.1136/bmjopen-2014007354

- Prepublication history for this paper is available online. To view these files please visit the journal online (http://dx.doi.org/10.1136/ bmjopen-2014-007354).

Received 3 December 2014 Revised 14 May 2015 Accepted 15 May 2015

CrossMark

For numbered affiliations see end of article.

Correspondence to Professor Rob Horne; r.horne@ucl.ac.uk

\section{ABSTRACT}

Objectives: The Necessity-Concerns Framework (NCF) posits that non-adherence to inhaled corticosteroids (ICS) in asthma is influenced by doubts about the necessity for ICS and concerns about their potential adverse effects. This feasibility study examined whether these beliefs could be changed by briefing asthma nurse specialists on ways of addressing necessity beliefs and concerns within consultations.

Design: Pre-post intervention study.

Setting: Secondary care.

Participants: Patients with a diagnosis of moderate to severe asthma who were prescribed daily ICS were recruited to either a hospital care group $(n=79 ; 71.0 \%$ female) or intervention group ( $n=57 ; 66.7 \%$ female). Intervention: Asthma nurse specialists attended a 1.5-day NCF briefing.

Primary and secondary outcome measures:

Beliefs about ICS (primary outcome) and self-reported adherence were measured preconsultation and 1 month postconsultation. Participants also rated their satisfaction with their consultations immediately after the consultation. Consultation recordings were coded to assess intervention delivery.

Results: After the NCF briefing, nurse specialists elicited and addressed beliefs about medicine more frequently. The frequency of using the NCF remained low, for example, open questions eliciting adherence were used in 0/59 hospital care versus 14/49 (28.6\%) intervention consultations. Doubts about personal necessity for, and concerns about, ICS were reduced at 1 month postbriefing $(p<0.05)$, but the intervention was not applied extensively enough to improve adherence.

Conclusions: The intervention changed nurse consultations, but not sufficiently enough to fully address non-adherence or adherence-related ICS beliefs (necessity and concerns). More effective techniques are needed to support nurse specialists and other practitioners to apply the intervention in hospital asthma review consultations.

\section{Strengths and limitations of this study}

- This study reports on the feasibility of delivering a theory-based intervention to modify beliefs within current models of asthma care (asthma nurse consultations).

- Recruitment and retention rates were high for this type of intervention, reducing potential bias in the data.

- Recordings of the study were used to test intervention fidelity robustly.

- As this was a pre-post study, confounders such as changes in nurses' consultations or patient outcomes due to external factors (eg, seasonal variation, receipt of other training) could not be controlled.

- Sample sizes were relatively small (especially of nurses), so we cannot be confident that these findings could extrapolate to nurses and patients at other clinics.

Daily inhaled corticosteroids (ICS) are the most effective method of controlling persistent asthma. ${ }^{1}{ }^{2}$ However, up to $60-70 \%$ of asthma patients do not take their ICS as prescribed, ${ }^{3}$ with non-adherence associated with increased exacerbations and mortality. ${ }^{4}{ }^{5}$ Addressing non-adherence is 1 of the 10 priorities in the Brussels Declaration for reducing the burden of asthma on individuals and society. ${ }^{1}{ }^{6}$ The reasons for nonadherence are complex, and can include barriers at the healthcare system level, for example, costs and accessibility of healthcare, factors associated with the healthcare provider, for example, whether they have the necessary knowledge and skills to support patients to adhere. ${ }^{7}$ At the individual level, these barriers can be summarised as a 
combination of perceptual factors (eg, beliefs about illness and treatment) and practical factors (eg, capacity and resources) influencing motivation and ability to adhere. $^{8}$

Studies conducted across long-term conditions including asthma have identified the Necessity-Concerns Framework (NCF) as a useful method for operationalising adherence-related beliefs. ${ }^{9-11}$ The framework posits that adherence to medication is influenced by patients' evaluation of treatment, specifically judgements of the personal need for treatment (necessity beliefs) relative to concerns about the potential negative effects (concerns). ${ }^{11}{ }^{12}$ Necessity beliefs are influenced by illness beliefs, treatment experiences and expectations; in symptom-free periods, patients with asthma may question their need for ICS ("no symptoms, no asthma'). ${ }^{8} 1314$ Concerns often extend beyond experienced side effects to issues including long-term harm and dependency. ${ }^{8}$ Beliefs may diverge from medical evidence but can have a 'common-sense' logic, for example, generalising oral high-dose corticosteroid side effects to low-dose ICS. ${ }^{13-15}$ Beliefs about specific medicines are related to general attitudes to pharmaceuticals and may be rooted in cultural norms. ${ }^{16} 17$

The NCF was proposed as an extension of Leventhal's Common-Sense Model. ${ }^{18}{ }^{19}$ In this model, internal information (eg, symptoms) and external information (eg, medical advice) are potentially threatening to patients and trigger cognitive and emotional responses in an attempt to manage the threat and its impact on self system. These responses can include evaluations (eg, of the likely consequences of an illness), emotions (eg, fear), and subsequent coping strategies (eg, healthcare seeking). The model recognises that these evaluations and coping strategies are themselves evaluated, and influence future responses to the threat. The NCF extended this model by suggesting that in addition to evaluations of the health threat, perceptions of treatments would also be important in determining treatment-related responses, for example, decisions to uptake and adhere to medication. ${ }^{19}$

Asthma guidelines recommend patient-centred review, including adherence, and that treatment beliefs are addressed. ${ }^{1}{ }^{20-22}$ Recent interventions have addressed treatment beliefs through brief telephone calls from pharmacists and text messages challenging/reinforcing medication and illness beliefs, ${ }^{23}{ }^{24}$ but it is unclear as to how to deliver NCF interventions within the existing models of asthma care. ${ }^{6825}$

In the UK, asthma review is often undertaken by asthma nurse specialists. ${ }^{1}$ In this feasibility study, as part of a Medical Research Council (MRC) guidance intervention development process, ${ }^{26}$ we examined the effect of an NCF briefing covering causes of non-adherence, and how to identify and address doubts about ICS necessity and concerns on asthma nurse specialists' consultations. We evaluated whether it resulted in more focus on adherence and beliefs about ICS within consultations, with subsequent improvements in patients' perceptions of ICS, adherence and satisfaction with consultations. We assessed two premises to evaluate the potential efficacy of the intervention: (1) ICS necessity beliefs and concerns predict adherence in this group and (2) that nurse specialists would elicit and address ICS beliefs more frequently postbriefing, indicating fidelity in delivery of the intervention.

\section{METHOD \\ Design}

This study was a pre-post intervention study. We selected this pre-post design for the pilot to enable us to compare nurses' consultations before and after their training. This was a pragmatic decision for the pilot because we did not have the resources to recruit and randomise nurses to receive the intervention training or not, which would have required large numbers of nurses and patients. We used systematic coding of audio recordings of consultations and validated questionnaires given to patients before consultation, immediately after consultation and at 1 month follow-up to assess consultations. We assessed a prebriefing (referred to as the 'hospital care' cohort below) cohort, gave nurse specialists the NCF briefing and then assessed a postbriefing cohort (the 'intervention' group; figure 1). The study was approved by the South East Multi-Centre Research Ethics Committee.

Participants were recruited and followed up between May 2005 and January 2006. Informed consent was obtained from patients and nurse specialists before participation. For the first 3 months, nurse specialists recruited patients into the hospital care group. Following this, they received their NCF training (see below), and then for a further 3 months they recruited additional patients into the intervention group. Patients completed four questionnaires (see figure 1). While waiting for their appointment, they completed a paper copy of the preconsultation ICS Questionnaire and, with the nurse, they completed the clinical assessment. At the end of their consultation, they were handed a paper Consultation Questionnaire with a stamped addressed envelope to complete and return immediately via post to the research team, not their nurse. Finally, the ICS Questionnaire and a stamped addressed envelope were sent to the participants 1 month after their appointment. If participants did not return either questionnaire, they were sent a reminder letter. An individual's questionnaire responses were not disclosed to nurse specialists.

\section{Participants}

Consecutive patients (intervention $\mathrm{n}=77$; hospital care $\mathrm{n}=98$ ) were included if they were aged 18 years or over and had moderate to severe asthma (as opposed to a mixed pattern). They were recruited by three experienced asthma nurse specialists (two other nurse specialists withdrew after being recruited into the study) from 


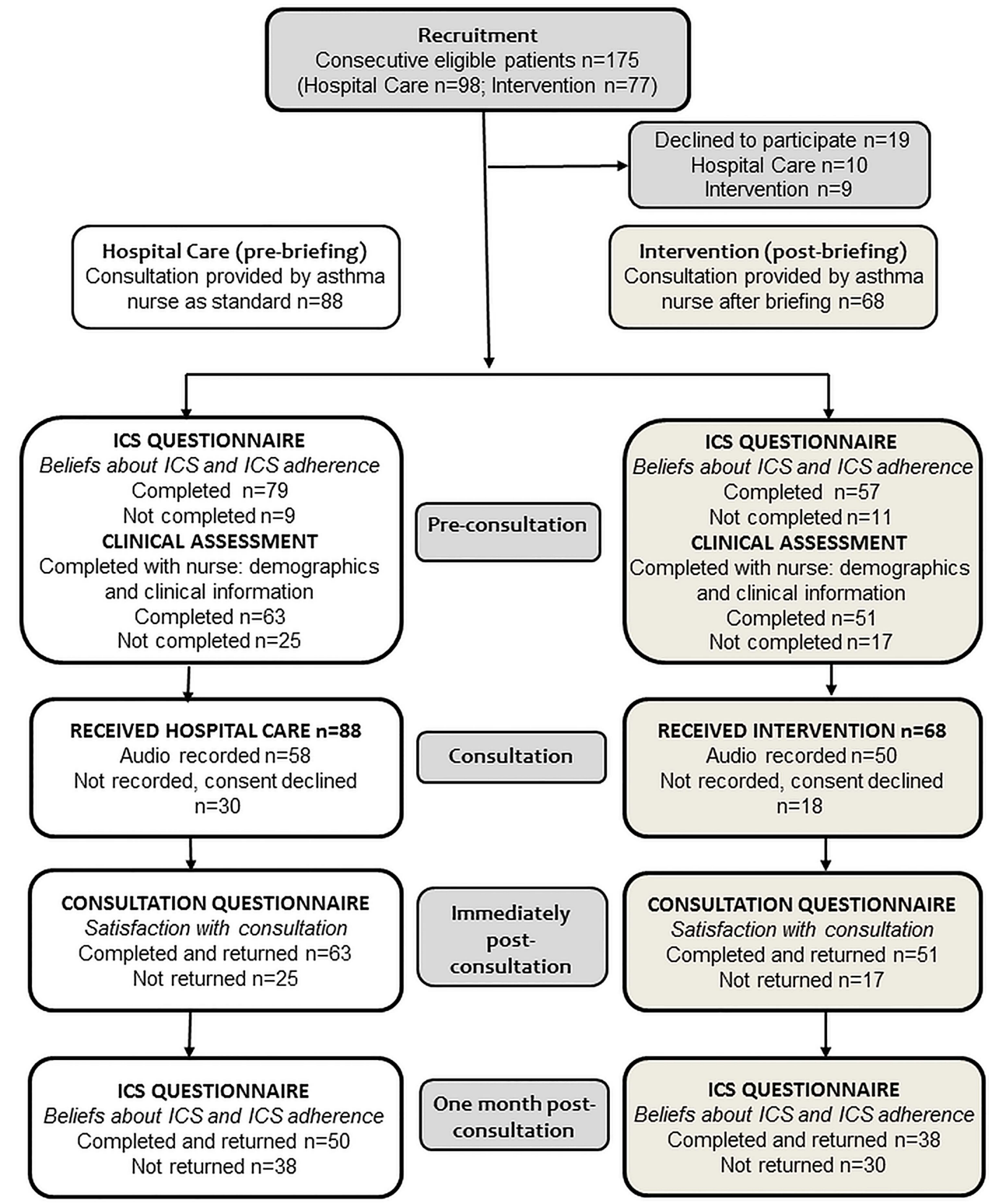

Figure 1 Procedure and questionnaire measures.

secondary/tertiary centres at Brighton General Hospital, Guy's Hospital (London) and the London Chest Hospital. In both groups, nearly $90 \%$ of eligible participants who were approached by the nurses consented to take part in the study (intervention: 88.7\%; hospital care: $89.6 \%$ ).

\section{Intervention}

The asthma nurse specialists delivered the intervention to participants. They were encouraged to target beliefs about asthma and ICS within their hospital practice and briefed on the rationale behind this approach over one full-day and one half-day sessions. We provided NCF manuals detailing adherence-related beliefs and guidelines for eliciting and addressing these beliefs including a consultation flow chart. The guidelines suggested three consultation components following National Institute for Health and Care Excellence (NICE) guidance: ${ }^{22}$ (1) communicating a no-blame approach to non-adherence to facilitate openness; (2) eliciting ICS necessity beliefs and concerns and (3) addressing doubts about ICS necessity beliefs and concerns (table 1). Separate manual pages were provided for reference during consultations.

\section{Measures}

The ICS Questionnaire contained two scales:

1. The Beliefs about Medicines Questionnaire Specific Asthma version ${ }^{13}$ measures beliefs about the personal need for ICS (9 items), for example, My health, at present, depends on this inhaler, and concerns about the potential adverse consequences of ICS (12 items), for example, I sometimes worry about the long term effects of this inhaler. Statements are endorsed on a Likert-type scale (range $1=$ strongly disagree to $5=$ strongly agree). We calculated mean item scores (range 1-5) to facilitate scale comparisons. ${ }^{27}$ The Necessity-Concerns 
Table 1 Intervention components

Intervention

Step 1: Provide a 'no-blame' space to report any non-adherence by prefacing the adherence discussion with an accepting statement

Step 2: Elicit patients' beliefs about their personal need for medication including in the absence of symptoms

AND

Elicit concerns about potential adverse effects of medication

Step 3: Address concerns or doubts about necessity using example rationales

Finally, address any other queries including practical issues

\section{Example}

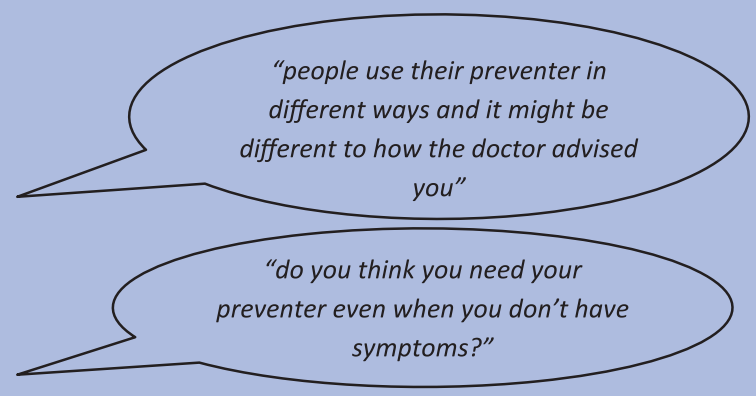

"people use their preventer in

different ways and it might be different to how the doctor advised

"do you think you need your symptoms?"

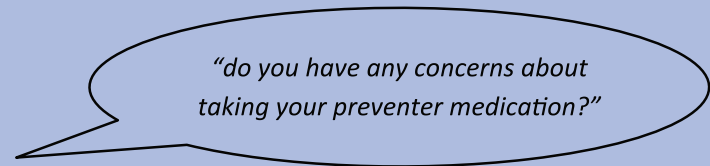

"you need preventer medication because asthma is permanent, caused by over-reactivity of the lungs and inflammation. Preventer medication works over time to reduce inflammation."

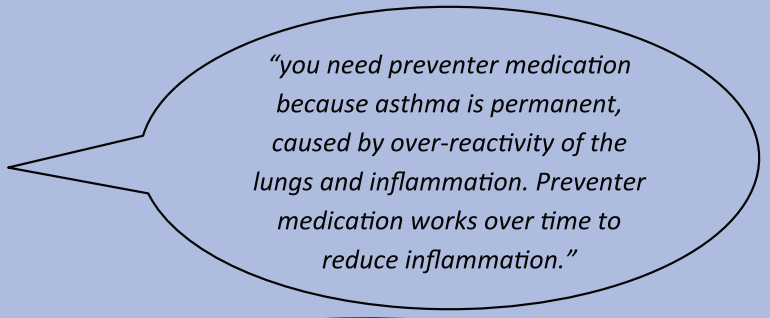

"can you tell me how you are using your spacer"
Differential (NCD) was calculated by subtracting the ICS concerns score from the ICS necessity score (range -4 to 4 ). It indicates patients' overall evaluation of $\mathrm{ICS} ;{ }^{23} 28{ }^{29}$ scores are positive if the perceived need for ICS is high relative to ICS concerns. The scale is validated for use in asthma samples and had acceptable reliability at baseline (necessity: Cronbach's $\alpha=0.83$; concerns: Cronbach's $\alpha=0.80){ }^{13}$

2. A brief four-item version of the Medication Adherence Report Scale (MARS) was used to measure self-reported adherence to ICS. Each item (eg, I forget to take my preventer inhaler) was rated on a Likert-type scale (range $5=$ never to 1 =always). ${ }^{27}$ Higher total scores (range 4-20) indicate higher adherence. Items were adapted from the original MARS to apply to asthma preventer medication, and one item, I take less than instructed, was omitted from the scale because it was felt by the research team to be ambiguous in the context of inhaled asthma treatment. Since previous studies have estimated that approximately a third of patients may not adhere to their medication, we compared the lowest third of participants on the MARS 'low adherers' $(\leq 16)$ with the highest two-thirds 'high adherers' $(>16) .{ }^{30}$ The MARS has been validated; ${ }^{31}$ reliability in the current sample was lower than in previous studies
(Cronbach's $\alpha=0.51$ ). Since this was a new version of the MARS, and the dichotomisation cut-off had not been validated, we conducted two additional sensitivity analyses, first, treating the MARS scores as continuous, and second, using a cut-off to compare people who reported any non-adherence (MARS scores $<20)$ to full adherence (MARS scores=20).

The Demographic and Clinical Information Questionnaire was completed with the asthma nurses. Patients reported their age, gender, age on leaving fulltime education, ethnicity and marital status. Patients also reported age of asthma onset, if they smoked currently, had relatives with asthma or any comorbid illnesses.

The Consultation Questionnaire contained two scales:

1. The Medical Interview Satisfaction Scale (MISS) assesses patient satisfaction with consultations. We omitted two items concerning diagnosis (all participants had an asthma diagnosis), leaving 19 items $^{32} 33$ rated on a seven-point Likert-type scale (1=very strongly disagree to $7=$ very strongly agree). Higher total scores indicate greater satisfaction (range 19-133, Cronbach's $\alpha=0.76$ ).

2. Satisfaction with intervention skills (MP5) was a new scale measuring perceptions of skills targeted by the intervention. It contains five items (figure 2), rated using the seven-point Likert-type scale used in the 


\begin{tabular}{|ll|}
\hline MP5 & \\
1. & The nurse gave me a clear understanding of why I need to use my preventer \\
2. The nurse helped me understand that asthma is long term and needs regular treatment \\
3. The nurse explained why I should use my preventer even when I don't have symptoms \\
4. The nurse helped me understand the effect preventer medication has on my body \\
5. The nurse provided me with a clear understanding of my asthma
\end{tabular}

Figure 2 Intervention-specific skills measure (MP5) items.

MISS. Higher total scores (range 5-35) indicate greater satisfaction with intervention skills. The scale has not been previously validated. It had good reliability, Cronbach's $\alpha=0.90$.

\section{Assessment of fidelity}

Consultations were audio recorded and transcribed. Two independent researchers, blind to whether consultations were prebriefing or postbriefing, rated transcriptions for intervention skills using a predefined schedule. For example, we assessed eliciting ICS concerns using Did the nurse elicit the patient's concerns about the medication? scored $0=$ =No'; 1='Asked indirectly'; 2='Yes-asked directly'. Responses were collapsed into those where both raters stated that the behaviour was unambiguously present, for example, coded 'Yes-asked directly'. Byrt's prevalence and bias-adjusted $\kappa$ (PABAK) was used to calculate agreement between raters, in addition to Cohen's $\kappa$, which is adversely affected when an outcome has low prevalence. ${ }^{34}$ Inter-rater agreement was adequate-good for eliciting concerns $(\kappa=0.76$, PABAK=0.80), asking about adherence using a no-blame frame $(\kappa=0.38$, PABAK $=0.58)$, addressing all concerns $(\kappa=0.53$, PABAK $=0.58)$ and addressing necessity beliefs using the manual $(\kappa=0.65, \mathrm{PABAK}=0.76)$. Agreement regarding whether necessity beliefs were elicited was low, $\kappa=0.17$ due to the low frequency of elicitations, $\mathrm{PABAK}=0.68$; however, raters agreed on $72 \%$ of interviews. Where one rater thought that the behaviour was present and another stated it was not, we erred on the side of caution when resolving these disagreements by assuming that the behaviour was not present. For example, where one rater thought that the nurse had asked about adherence using a no-blame frame, whereas the other rater did not, we coded this as not being present.

\section{Analysis}

Sample size calculation: As this was a feasibility study, we did not perform a sample size calculation.

Missing data: Mean ICS necessity and concerns values were calculated. Where four or more values were missing, the whole scale was treated as missing; where three or fewer values were missing, the mean of the completed items was used. Analyses were repeated using a data set derived using multiple imputation of missing data. The pattern of results was similar, so original results are reported here.
Hypothesis testing: Paired samples within group t tests were used to compare ICS beliefs and adherence at baseline and follow-up in both groups. Between-groups independent samples $t$ tests and $\chi^{2}$ were performed to compare the hospital care with the intervention group on demographic and clinical variables, nurses' consultation behaviour and consultation satisfaction; to compare high and low adherers' ICS beliefs; and to compare participants who completed follow-up with those who did not, on demographic and clinical factors, baseline beliefs and adherence. Repeated measures analyses of variance (ANOVAs) were used to test for differences in changes in beliefs between baseline and follow-up between groups. Non-parametric correlations were used to test for associations between beliefs and self-reported adherence at baseline, when adherence was treated as a continuous variable.

\section{RESULTS}

The intervention and hospital care groups did not differ significantly (all $\mathrm{p}>0.05$ ) on the demographic and clinical variables (table 2) at baseline. Forty-eight participants $(35.3 \%)$ completed the preconsultation ICS Questionnaire but did not complete the follow-up ICS Questionnaire (see figure 1 for details of the flow of participants through the study). Participants who did not continue were younger (mean age $=37.37$ years, $\mathrm{SD}=14.61$ years) than those who completed the follow-up survey (mean age $=50.58$ years, $\mathrm{SD}=16.27$ years, $\mathrm{p}<0.001$ ), less likely to report white ethnicity; $17(17.9 \%)$ white participants and $10(58.8 \%)$ other ethnicity participants did not complete the follow-up. There were no other significant differences between those who completed and those who did not.

\section{Intervention effects}

Intervention participants had fewer doubts about their personal need for, and concerns about, ICS at 1 month follow-up, relative to baseline. The NCD at follow-up increased significantly, indicating increased perceptions of ICS necessity relative to concerns about ICS postconsultation (see table 3 ).

In the hospital care group, patients' necessity and concern beliefs and NCD score did not significantly change after consultation. Repeated-measures ANOVAs with the between-subjects factor of group (intervention or hospital care) and within-subjects factor of time 
Table 2 Baseline demographic and clinical characteristics

\begin{tabular}{|c|c|c|}
\hline & $\begin{array}{l}\text { Hospital } \\
\text { care }(n=63) \\
\text { Mean }(S D)\end{array}$ & $\begin{array}{l}\text { Intervention } \\
(\mathrm{n}=51) \\
\text { Mean (SD) } \\
\end{array}$ \\
\hline Age (years) & $46.7(17.2)^{\star}$ & $47.8(16.6)$ \\
\hline Age left education (years) & $19.5(8.74)^{\dagger}$ & $18.6(8.43) \ddagger$ \\
\hline \multirow{2}{*}{$\begin{array}{l}\text { Age of asthma onset } \\
\text { (years) }\end{array}$} & $23.7(19.7) \S$ & $22.1(19.7) \uparrow$ \\
\hline & n (\%) & n (\%) \\
\hline Female & $44(71.0)^{*}$ & $34(66.7)$ \\
\hline White & $53(85.5)^{\star}$ & $43(84.3)$ \\
\hline Married & $30(49.2) \rrbracket$ & $26(52.0)^{*}$ \\
\hline Employed FT/PT & $30(48.4)^{\star}$ & $26(52.0)^{\star}$ \\
\hline Report comorbid illness & $34(54.8)^{\star}$ & 21 (42.9)ף \\
\hline Currently non-smoker & 55 (90.2) П & 45 (88.2) \\
\hline Family history of asthma & $36(59.0) \emptyset$ & $33(66.0)^{*}$ \\
\hline \multicolumn{3}{|c|}{$\begin{array}{l}\text { *One participant missing data. } \\
\text { †Eight participants missing data. } \\
\text { ‡Seven participants missing data. } \\
\text { §Three participants missing data. } \\
\text { १Two participants missing data. } \\
\text { FT/PT, full time/part time. }\end{array}$} \\
\hline
\end{tabular}

(baseline or follow-up) for ICS necessity, ICS concerns and the NCD were used to investigate whether changes in beliefs were larger in the intervention group than in the hospital care group. There was a significant main effect of time for ICS concerns $F(1,82)=4.42, p=0.04$ and the $\operatorname{NCD}(1,82)=11.48, \mathrm{p}<0.01$, but not for ICS necessity $\mathrm{F}(1,82)=3.26, \mathrm{p}=0.07$. There were no significant main effects of group for ICS necessity $\mathrm{F}(1,86)=0.16, \mathrm{p}=0.69$, ICS concerns $\mathrm{F}(1,82)=1.41, \mathrm{p}=0.24$ or $\mathrm{NCD} \mathrm{F}(1,82)$ $=0.72, p=0.40$. Interaction terms indicated that changes in beliefs were not significantly larger in the intervention group than in the hospital care group for ICS

Table 3 ICS beliefs at baseline and at 1 month follow-up

$\begin{array}{ll}\text { Baseline, } & \text { Follow-up, } \\ \text { Mean (SD) } & \text { Mean (SD) }\end{array}$

\begin{tabular}{|c|c|c|c|}
\hline \multicolumn{4}{|c|}{ Intervention group $(n=38)$} \\
\hline ICS necessity & $3.89(0.63)$ & $4.04(0.55)$ & $\begin{array}{l}t(37)=2.25 \\
p=0.03\end{array}$ \\
\hline ICS concerns & $2.54(0.59)$ & $2.40(0.65)$ & $\begin{array}{l}t(37)=2.22 \\
p=0.03\end{array}$ \\
\hline NCD & $1.35(0.92)$ & $1.64(0.90)$ & $\begin{array}{l}t(37)=2.81 \\
p<0.01\end{array}$ \\
\hline \multicolumn{4}{|c|}{ Hospital care group $(n=50)$} \\
\hline ICS necessity & $3.86(0.72)$ & $3.97(0.68)$ & $\begin{array}{l}t(49)=0.96 \\
p=0.34\end{array}$ \\
\hline ICS concerns* & $2.35(0.55)$ & $2.31(0.44)$ & $\begin{array}{l}t(45)=0.70 \\
p=0.49\end{array}$ \\
\hline NCD* & $1.57(0.86)$ & $1.71(0.72)$ & $\begin{array}{l}t(45)=1.84 \\
p=0.07\end{array}$ \\
\hline
\end{tabular}

necessity $\mathrm{F}(1,86)=0.08, \mathrm{p}=0.77$, ICS concerns $\mathrm{F}(1,82)$ $=1.37, \mathrm{p}=0.25$ or the NCD $\mathrm{F}(1,82)=1.18, \mathrm{p}=0.28$.

No significant change in reported adherence to ICS between baseline and follow-up was found in either the intervention group, $\mathrm{z}=0.63, \mathrm{p}=0.53$ or hospital care group, $\mathrm{z}=0.16, \mathrm{p}=0.87$. Median MARS scores were similar for both groups (intervention group: baseline median=17.5, follow-up median=18.0; hospital care group: baseline median=18.0, follow-up median=18.0. At follow-up, 10/37 $(27.0 \%)$ intervention patients were low adherers relative to $9 / 46(19.6 \%)$ hospital care patients, $\chi^{2}(1, n=83)=0.647, p=0.44$. In the sensitivity analyses, the same pattern (no change in adherence) was also seen for participants who reported full adherence $($ MARS=20) to participants who reported any nonadherence $(\mathrm{MARS}<20)$.

Both groups reported high satisfaction with nurse specialists' generic consultation skills and interventionspecific consultation skills (MISS: intervention $\mathrm{m}=110.65$, $\mathrm{SD}=18.84$, hospital care $\mathrm{m}=107.16, \mathrm{SD}=16.95, \mathrm{t}(110)$ $=1.03, \mathrm{p}=0.31$; MP5: intervention $\mathrm{m}=28.82 \mathrm{SD}=5.41$, hospital care $\mathrm{m}=28.22, \mathrm{SD}=5.33, \mathrm{t}(107)=0.58, \mathrm{p}=0.56)$.

\section{Intervention premises}

At baseline, low adherers had more doubts about their need for ICS, more concerns about ICS medication and a significantly greater NCD score than high adherers ( $p<0.05$, table 4 and figure 3$)$. The same pattern was seen if participants who reported full adherence $(n=33)$ were compared with those who reported any nonadherence $(n=97)$. If continuous MARS scores were used, higher baseline adherence scores were associated with fewer ICS concerns in non-parametric correlations $(r(130)=-0.30, p<0.001)$, higher belief in personal need for ICS $(r(130)=0.28, p=0.001)$ and a greater NCD score $(r(130)=0.37, p<0.001)$. We assessed whether nurse specialists' consultations changed postbriefing for each step (eliciting non-adherence using a 'no-blame' frame, eliciting beliefs, addressing beliefs using a commonsense rationale).

Nurse specialists asked an open question about adherence in 0 hospital care consultations versus 14 (28.6\%)

Table 4 Beliefs about ICS across high and low adherers

\begin{tabular}{|c|c|c|c|}
\hline & $\begin{array}{l}\text { High } \\
\text { adherers } \\
(n=88) \text {, } \\
\text { Mean (SD) }\end{array}$ & $\begin{array}{l}\text { Low } \\
\text { adherers } \\
(n=42) \text {, } \\
\text { Mean (SD) }\end{array}$ & \\
\hline ICS necessity & $3.95(0.53)$ & $3.61(0.73)$ & $\begin{array}{l}t(128)=3.01, \\
p<0.01\end{array}$ \\
\hline ICS concerns & $2.44(0.55)$ & $2.74(0.55)$ & $\begin{array}{l}t(128)=2.82, \\
p<0.01\end{array}$ \\
\hline ICS NCD & $1.51(0.87)$ & $0.88(0.93)$ & $\begin{array}{l}\mathrm{t}(128)=3.81, \\
\mathrm{p}<0.001\end{array}$ \\
\hline
\end{tabular}




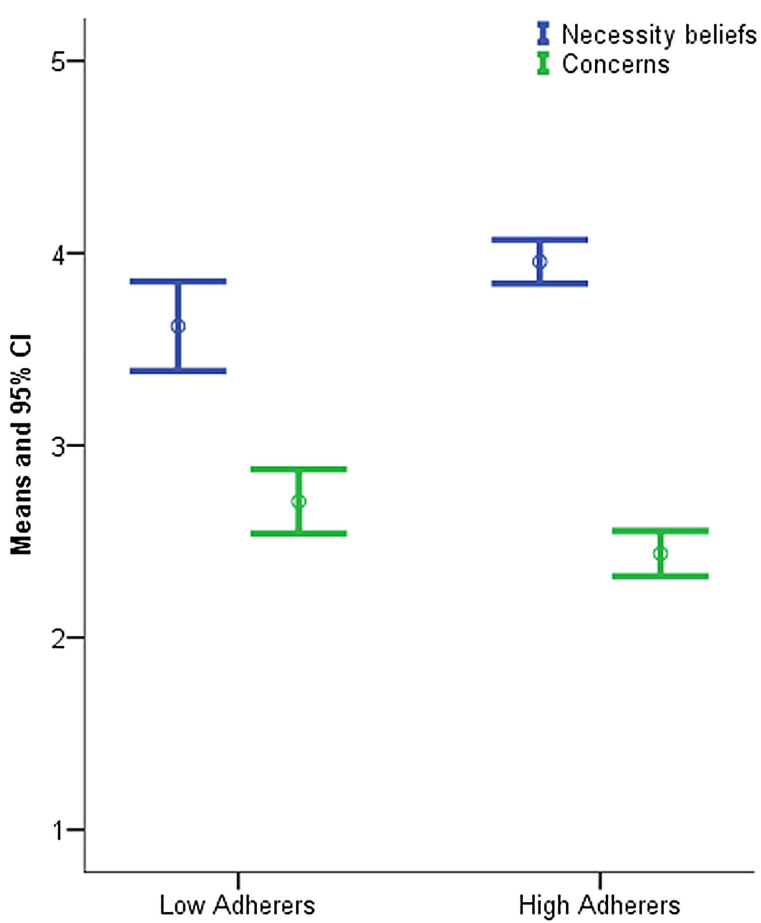

Adherence based on dichotomised MARS score

Figure 3 Inhaled corticosteroids (ICS) necessity beliefs and ICS concerns in patients with high and low adherence, at baseline.

intervention consultations, $\chi^{2}(1, \mathrm{n}=108)=19.37, \mathrm{p}<0.001$, demonstrating a significant change in consultation style. However, adherence was not elicited in this way in 35/49 $\mathbf{7} 72.4 \%)$ consultations postbriefing. Nurse specialists elicited patients' ICS beliefs more frequently after the briefing intervention. Beliefs about ICS necessity in the absence of symptoms were elicited in 9 (18.4\%) intervention consultations versus 0 hospital care consultations, $\chi^{2}(1, \mathrm{n}=108)=11.63, \mathrm{p}=0.001$. ICS concerns were elicited in $31(63.3 \%)$ intervention consultations versus $1(1.7 \%)$ hospital care consultations, $\chi^{2}(1, \mathrm{n}=108)$ $=48.67, \mathrm{p}<0.001$. Nurse specialists communicated the necessity of taking ICS in the absence of symptoms in 18 $(30.5 \%)$ hospital care consultations versus 21 (43.8\%) intervention consultations, $\chi^{2}(1, \mathrm{n}=107)=2.00, \mathrm{p}=0.13$. Nurse specialists provided information to address patients' stated concerns about the potential adverse consequences of ICS on $3(5.1 \%)$ occasions prebriefing and $17(34.7 \%)$ occasions postbriefing, $\chi^{2}(1, n=108)$ $=15.55, \mathrm{p}<0.001$. Despite this increase, in the majority $(32 / 49)$ of intervention consultations, ICS concerns were not clearly addressed.

\section{DISCUSSION}

This feasibility study was part of an NCF intervention development process, following MRC guidance. ${ }^{26}$ We tested the feasibility of briefing asthma nurse specialists to support adherence by eliciting and addressing adherence-related ICS necessity beliefs and concerns during hospital consultations; examined process variables to evaluate why the intervention did/did not work; indexed fidelity of delivery; and measured patient satisfaction. At follow-up, intervention participants' doubts about ICS necessity and concerns decreased significantly, indicating that adherence-related beliefs are amenable to change. This change coincided with an increase in the frequency with which nurse specialists applied NCF components. This approach was not used consistently, meaning that the intervention effect across the groups was small.

As predicted, ICS necessity beliefs and concerns were related to reported adherence, confirming the premise that addressing these might improve adherence and consistency with NICE guidance ${ }^{8}{ }^{22}$ and a recent meta-analysis. ${ }^{11}$ We tested the premise that the briefing would change nurse specialists' consultation behaviour. Postbriefing, nurse specialists used a 'no-blame' frame to ask about adherence in $28.6 \%$ of consultations, elicited doubts about the necessity of ICS in $18.6 \%$ of consultations, and elicited concerns about the adverse effects of ICS in $63.3 \%$ of consultations. Prebriefing, concerns were elicited once, doubts about ICS necessity were not elicited, and nor was an open, 'no-blame' question regarding adherence used. Nurse specialists may need further support to facilitate honest and open discussions of nonadherence as recommended by NICE. ${ }^{22}$ However, despite these postbriefing changes, concerns about the adverse effects and doubts about the need for ICS remained unaddressed in most consultations. Discussing but not addressing medication concerns and doubts may reduce the efficacy of the NCF approach. For example, providing a rationale for ICS without eliciting necessity beliefs may prevent appropriate tailoring of the rationale; therefore, we may have demonstrated greater efficacy if fidelity of intervention delivery been high.

In the intervention group, doubts about ICS necessity and ICS concerns were reduced at 1 month after the nurse consultation suggesting that after the briefing, nurse specialists were better able to reassure their patients regarding their asthma treatment. The lack of change in adherence is perhaps not surprising given the high baseline adherence, risking a ceiling effect, and the low fidelity of intervention delivery achieved. We assessed patients' satisfaction with the consultations. Satisfaction scores were high, prebriefing and postbriefing, for both generic skills measured by the MISS and intervention-specific skills measured by the MP5, indicating that patients were typically very satisfied with the nurse specialists' consultations.

This was a feasibility study, and so was not designed or powered to detect a change in intervention outcomes relative to hospital care, explore heterogeneity across sites or between nurses, or evaluate uncontrolled factors (eg, increased experience of the nurse specialists, or change in recruitment style after the briefing resulting in selection bias). We used self-report rather than more objective adherence measures (eg, electronic monitoring). We also relied on the nurses delivering the intervention to recruit participants and hand out 
questionnaires. It appears that as the demands of running busy clinics were understandably prioritised above collecting research data, this approach may have resulted in a higher than anticipated level of missing data (eg, for the completion of clinical assessment). A large randomised controlled trial with specifically allocated research assistance and additional adherence measures may resolve these limitations; however, further intervention development is needed. Specifically, given the possible ceiling effects, interventions should target non-adherent patients or those whose ICS perceptions indicate risk of non-adherence. New technologies for supporting consultations in real time and structured NCF scripts or aids may facilitate the identification of these patients, and increase fidelity to the intervention. Training nurse specialists more extensively, for example, over more than two sessions, or changing the briefing content to include techniques such as motivational interviewing, where patients are encouraged to explore ambivalence to increase motivation, ${ }^{35}{ }^{36}$ or cognitive behavioural methods, for example, problem solving, cognitive restructuring, ${ }^{37}$ may increase efficacy.

This study confirms the importance of eliciting and addressing patients' ICS necessity beliefs and concerns in relation to non-adherence. The briefing led to increased necessity beliefs and reduced concerns, but the NCF was not implemented consistently, so the effects were small. The NCF intervention is promising, but alternative methods, such as training for nurse specialists and new technologies, should be explored.

\author{
Author affiliations \\ ${ }^{1}$ Centre for Behavioural Medicine, UCL School of Pharmacy, London, UK \\ ${ }^{2}$ London Chest Hospital, Barts Health NHS Trust, London, UK \\ ${ }^{3}$ Florence Nightingale School of Nursing and Midwifery, Kings College, \\ London, UK \\ ${ }^{4}$ Department of Respiratory Medicine, Royal Sussex County Hospital, \\ Brighton, UK \\ ${ }^{5}$ Asthma UK 18, London, UK \\ ${ }^{6}$ Institute of Pharmaceutical Sciences \& Institute of Psychiatry, Kings College \\ London, London, UK
}

Acknowledgements The authors would like to thank the nurse specialists for their involvement in this study, as well as all the participants for giving up their time to take part in this study. They would also like to thank Dr Li Wei for her advice on the statistical analysis of this data, and Rhian Parham and Jenny Hitchens for their assistance with the study.

Contributors $\mathrm{RH}$ is the guarantor of the paper. $\mathrm{RH}, \mathrm{MB}, \mathrm{NB}, \mathrm{CP} \mathrm{JH}, \mathrm{AW}$ and JW contributed to the design and conduct of the study. SCEC and RH contributed to the data analysis. All others contributed to the writing of the manuscript.

Funding This research was funded by an educational grant from Asthma UK. RH was supported by an NIHR Senior Investigator award, and SCEC's time was supported by the NIHR.

Competing interests $\mathrm{RH}$ reports grants from Asthma UK, grants from NIHR Senior Investigator Award and during the conduct of the study; and personal fees from Spoonful of Sugar/GSK/Astra Zeneca, outside the submitted work. SCEC reports support from Asthma UK, and indirectly through an NIHR Senior Investigator Award, during the conduct of the study; and personal fees from Nesta/Spoonful of Sugar/GSK/Astra Zeneca, outside the submitted work. NB reports other funding from GSK and is employed by GSK.
Ethics approval South East Multi-Centre Research Ethics Committee.

Provenance and peer review Not commissioned; externally peer reviewed.

Data sharing statement No additional data are available.

Open Access This is an Open Access article distributed in accordance with the Creative Commons Attribution Non Commercial (CC BY-NC 4.0) license, which permits others to distribute, remix, adapt, build upon this work noncommercially, and license their derivative works on different terms, provided the original work is properly cited and the use is non-commercial. See: http:// creativecommons.org/licenses/by-nc/4.0/

\section{REFERENCES}

1. British Thoracic Society-Scottish Intercollegiate Guidelines Network B-S. British guideline on the management of asthma. Healthcare Improvement Scotland. 2008 (revised 2012).

2. National Asthma Education and Prevention Program. Expert panel report 3: guidelines for the diagnosis and management of asthma-summary report 2007. J Allergy Clin Immunol 2007;120(5 Suppl):S94-138.

3. Sumino K, Cabana MD. Medication adherence in asthma patients. Curr Opin Pulm Med 2013;19:49-53.

4. Williams LK, Peterson EL, Wells $\mathrm{K}$, et al. Quantifying the proportion of severe asthma exacerbations attributable to inhaled corticosteroid nonadherence. J Allergy Clin Immunol 2011;128:1185-91.

5. Suissa S, Ernst $P$, Benayoun $S$, et al. Low-dose inhaled corticosteroids and the prevention of death from asthma. $N$ Engl $J$ Med 2000;343:332-6.

6. Holgate $\mathrm{S}$, Bisgaard $\mathrm{H}$, Bjermer $\mathrm{L}$, et al. The Brussels declaration: the need for change in asthma management. Eur Respir $J$ 2008;32:1433-42.

7. Dima AL, Hernandez G, Cunillera O, et al. Asthma inhaler adherence determinants in adults: systematic review of observational data. Eur Respir J 2014:45:994-1018.

8. Horne R. Compliance, adherence, and concordance: implications for asthma treatment. Chest 2006;130(1 Suppl):65S-72S.

9. Sofianou A, Martynenko M, Wolf MS, et al. Asthma beliefs are associated with medication adherence in older asthmatics. J Gen Intern Med 2013;28:67-73.

10. Phatak HM, Thomas J III. Relationships between beliefs about medications and nonadherence to prescribed chronic medications. Ann Pharmacother 2006:40:1737-42.

11. Horne R, Chapman SCE, Parham R, et al. Understanding patients' adherence-related beliefs about medicines prescribed for long-term conditions: a meta-analytic review of the Necessity-Concerns Framework. PLOS ONE 2013;8:e80633.

12. Horne R, Weinman J, Barber N, et al. Concordance, adherence and compliance in medicine taking. Report for the National Co-ordinating Centre for NHS Service Delivery and Organisation R \& D. 2005. 2005.

13. Horne R, Weinman J. Self regulation and self management in asthma: exploring the role of illness perceptions and treatment beliefs in explaining non-adherence to preventer medication. Psychol Health 2002;17:17-32.

14. Halm EA, Mora P, Leventhal $H$. No symptoms, no asthma: the acute episodic disease belief is associated with poor self-management among inner-city adults with persistent asthma. Chest 2006:129:573-80.

15. Clatworthy J, Price D, Ryan D, et al. The value of self-report assessment of adherence, rhinitis and smoking in relation to asthma control. Prim Care Respir J 2009;18:300-5.

16. Kumar K, Gordon C, Barry R, et al. 'It's like taking poison to kill poison but I have to get better': a qualitative study of beliefs about medicines in rheumatoid arthritis and systemic lupus erythematosus patients of South Asian origin. Lupus 2011;20:837-44.

17. Horne R, Graupner L, Frost S, et al. Medicine in a multi-cultural society: the effect of cultural background on beliefs about medications. Soc Sci Med 2004;59:1307-13.

18. Cameron LD, Leventhal $\mathrm{H}$. The self-regulation of health and illness behaviour. Psychology Press, 2003.

19. Horne R. Treatment perceptions and self-regulation. The selfregulation of health and illness behaviour. London: Routledge, 2003:138-53.

20. Griffiths CJ. Consultations for asthma: will greater patient involvement deliver better health? Thorax 2005;60:177-8.

21. Horne R, Price D, Cleland J, et al. Can asthma control be improved by understanding the patient's perspective? BMC Pulmon Med 2007;7:8.

22. National Institute for Health and Clinical Excellence, ed. Medicines adherence: involving patients in decisions about prescribed medicines and supporting adherence. 2009. 
23. Clifford $\mathrm{S}$, Barber N, Elliott R, et al. Patient-centred advice is effective in improving adherence to medicines. Pharm World Sci 2006;28:165-70.

24. Petrie KJ, Perry K, Broadbent E, et al. A text message programme designed to modify patients' illness and treatment beliefs improves self-reported adherence to asthma preventer medication. Br J Health Psychol 2012;17:74-84.

25. Heaney LG, Horne R. Non-adherence in difficult asthma: time to take it seriously. Thorax 2012;67:268-70.

26. Craig P, Dieppe P, Macintyre S, et al. Developing and evaluating complex interventions: the new Medical Research Council guidance. BMJ 2008;337.

27. Horne R, Weinman J, Hankins M. The beliefs about medicines questionnaire: the development and evaluation of a new method for assessing the cognitive representation of medication. Psychol Health 1999;14:1-24.

28. Horne R, Weinman J. Patients' beliefs about prescribed medicines and their role in adherence to treatment in chronic physical illness. $J$ Psychosom Res 1999;47:555-67.

29. Horne R, Buick D, Fisher M, et al. Doubts about necessity and concerns about adverse effects: identifying the types of beliefs that are associated with non-adherence to HAART. Int J STD AIDS 2004;15:38-44.
30. Sabaté E. Adherence to long-term therapies: evidence for action. World Health Organization, 2003.

31. Cohen JL, Mann DM, Wisnivesky JP, et al. Assessing the validity of self-reported medication adherence among inner-city asthmatic adults: the Medication Adherence Report Scale for Asthma. Ann Allergy Asthma Immunol 2009;103:325-31.

32. Meakin R, Weinman J. The 'Medical Interview Satisfaction Scale' (MISS-21) adapted for British general practice. Fam Prac 2002;19:257-63.

33. Wolf MH, Putnam SM, James SA, et al. The medical interview satisfaction scale: development of a scale to measure patient perceptions of physician behavior. J Behav Med 1978;1:391-401.

34. Byrt T, Bishop J, Carlin JB. Bias, prevalence and kappa. J Clin Epidemiol 1993;46:423-9.

35. Miller WR, Rollnick S. Motivational interviewing: preparing people for change. Guilford press, 2002.

36. Martins RK, McNeil DW. Review of motivational interviewing in promoting health behaviors. Clin Psychol Rev 2009;29:283-93.

37. Safren SA, Gonzalez JS, Wexler DJ, et al. A randomized controlled trial of cognitive behavioral therapy for adherence and depression (CBT-AD) in patients with uncontrolled type 2 diabetes. Diabetes Care 2013;37:625-33. 